GA-A22070

\title{
PLASMA ROTATION \\ IN THE PRESENCE OF \\ RADIO FREQUENCY FIELDS
}

\author{
by \\ Y.R. LIN-LIU, V.S. CHAN, and S.C. CHIU
}

JUNE 1995

DISTRIBUTION OF THIS DOCUMENT IS UNLIMITED 


\section{DISCLAIMER}

This report was prepared as an account of work sponsored by an agency of the United States Government. Neither the United States Government nor any agency thereof, nor any of their employees, makes any warranty, express or implied, or assumes any legal liability or responsibility for the accuracy, completeness, or usefulness of any information, apparatus, product, or process disclosed, or represents that its use would not infringe privately owned rights. Reference herein to any specific commercial product, process, or service by trade name, trademark, manufacturer, or otherwise, does not necessarily constitute or imply its endorsement, recommendation, or favoring by the United States Government or any agency thereof. The views and opinions of authors expressed herein do not necessarily state or reflect those of the United States Government or any agency thereof. 


\section{DISCLAIMER}

Portions of this document may be illegible in electronic image products. Images are produced from the best available original document. 


\title{
PLASMA ROTATION \\ IN THE PRESENCE OF \\ RADIO FREQUENCY FIELDS
}

\author{
by \\ Y.R. LIN-LIU, V.S. CHAN, and S.C. CHIU
}

This is a preprint of a paper presented at the 11th Topical Conference on Radio Frequency Power in Plasmas, May 17-19, 1995, Palm Springs, California, and to be printed in the Proceedings.

\author{
Work supported by \\ U.S. Department of Energy \\ Contract DE-AC03-89ER51114
}

GENERAL ATOMICS PROJECT 3466

JUNE 1995

DISTRIBUTION OF THIS DOCUMENT IS UNLIMITED 


\title{
Plasma Rotation in the Presence of Radio Frequency Fields
}

\author{
Y.R. Lin-Liu, V.S. Chan, and S.C. Chiu
}

Genenal Atomics, San Diego, California 92186

\begin{abstract}
A combined moment and kinetic approach is developed to consider rf effects on plasma rotation. Appropriate relaxation times for driven toroidal and poloidal rotation are discussed.
\end{abstract}

\section{INTRODUCTION}

Plasma rotation in tokamaks suppresses low- $n$ pressure-driven magnetohydrodynamic (MHD) instabilities and is helpful in increasing the betalimit [1]. Shear in this rotation, either toroidal or poloidal, is thought to suppress turbulence and allow improvement in energy confinement [2]. Plasma rotation in present-day tokamaks is driven by neutral beam injection (NBI), which might not be used in a tokamak reactor. Moreover, the prospect for fusion-produced alpha particles to drive a significant amount of plasma rotation has recently been shown to be remote by Rosenbluth and Hinton [3]. Therefore, it is of interest to consider rf effects on plasma rotation. In this work, we incorporate the concept of $\mathrm{rf}$ forces previously proposed by two of us [4] in the theoretical formulation of Rosenbluth and Hinton for considering rf-induced rotation in toroidal geometry. In particular, we will discuss appropriate relaxation times of the plasma response to if rotation drive in the toroidal and poloidal directions.

\section{RF FORCES AND MOMENT APPROACH}

The concept of if forces has been advanced to give a unified description of resonant and nonresonant interactions of the plasma with the $\mathrm{rf}$ fields. In the context of a quasilinear theory [4], the rf force is shown to be 


$$
\begin{aligned}
& \vec{F}_{\alpha}^{\mathrm{rf}}=m_{\alpha} \int d^{3} v \vec{v} Q_{\alpha}^{\mathrm{rf}}, \\
& Q_{\alpha}^{\mathrm{rf}}=\left\langle\vec{\nabla}_{v} \cdot\left[\frac{q_{\alpha}}{m_{\alpha}}\left(\vec{E}_{1}+\vec{v} \times \vec{B}_{1}\right) f_{1 \alpha}\right]\right\rangle_{\theta} .
\end{aligned}
$$

Here, $\vec{E}_{1}$ and $\vec{B}_{1}$ denote the rf fields, $f_{1 \alpha}$ the fast oscillating part of the particle distribution of species $\alpha=\mathrm{i}$ or $\mathrm{e}$, and $\langle\ldots\rangle_{\theta}$ the gyrophase average. Neglecting electron inertia, the momentum equation in the presence of of fields is

$$
n_{\mathrm{i}} m_{\mathrm{i}}\left(\frac{\partial \vec{u}}{\partial t}+\vec{u} \cdot \vec{\nabla} \vec{u}\right)=-\vec{\nabla} p-\vec{\nabla} \cdot \stackrel{\leftrightarrow}{\Pi}+\vec{\jmath} \times \vec{B}+\vec{F}^{\mathrm{rf}}
$$

where $\vec{u}$ is fluid velocity, $p=\sum_{\alpha} p_{\alpha}$ is total plasma pressure, and $\stackrel{\leftrightarrow}{\Pi}$ denotes the traceless stress tensor. With the assumption of incompressibility, the general expression for $\vec{u}$ in toroidal geometry is

$$
\vec{u}=K(\psi) \vec{B}+G(\psi) R \widehat{e}_{\phi},
$$

where $K(\psi)$ and $G(\psi)$ are two flux-surface quantities. Note that the poloidal flow function is proportional to the poloidal velocity, i.e., $u_{\mathrm{p}}=K(\psi) B_{\mathrm{p}}$, and $G(\psi)$ is proportional to the perpendicular velocity.

It is convenient to consider the flux-surface average of toroidal and parallel components of Eq. (3),

$$
\begin{aligned}
& n_{\mathrm{i}} m_{\mathrm{i}} \frac{\partial\left\langle R u_{\phi}\right\rangle}{\partial t}=\langle\vec{\jmath} \cdot \vec{\nabla} \psi\rangle+\left\langle R \widehat{e}_{\phi} \cdot \vec{F}^{\mathrm{rf}}\right\rangle-\left\langle R \widehat{e}_{\phi} \cdot \vec{\nabla} \cdot \stackrel{\leftrightarrow}{\Pi}\right\rangle \\
& n_{\mathrm{i}} m_{\mathrm{i}} \frac{\partial\langle\vec{B} \cdot \vec{u}\rangle}{\partial t}=\left\langle\vec{B} \cdot \vec{F}^{\mathrm{rf}}\right\rangle-\langle\vec{B} \cdot \vec{\nabla} \cdot \stackrel{\leftrightarrow}{\Pi}\rangle
\end{aligned}
$$

The radial current $\langle\vec{\jmath} \cdot \vec{\nabla} \psi\rangle$ is related to the radial electric field by the Maxwell equation

$$
\frac{\partial}{\partial t}\langle\vec{E} \cdot \vec{\nabla} \psi\rangle+\mu_{0}\left\langle\left(\vec{\jmath}+\vec{\jmath}^{\text {ex }}\right) \cdot \vec{\nabla} \psi\right\rangle=0
$$

where $\vec{\jmath}^{\text {ex }}$ denotes some external current source [5]. In the absence of an external current source and in steady-state $\langle\vec{\jmath} \cdot \vec{\nabla} \psi\rangle=\left\langle\vec{\jmath}^{\text {ex }} \cdot \vec{\nabla} \psi\right\rangle=0$.

The steady-state flow can be determined by balancing the force and the stress tensor, if the latter is assumed to be proportional to the flow velocity. One plausible assumption is that $\left\langle R \widehat{e}_{\phi} \cdot \vec{\nabla} \cdot \stackrel{\leftrightarrow}{\Pi}\right\rangle=\tau_{\phi}^{-1} n_{\mathrm{i}} m_{\mathrm{i}}\left\langle u_{\phi} R\right\rangle$, where $\tau_{\phi}$ is identified as the confinement time for the toroidal angular momentum. It 
is generally believed that $\tau_{\phi} \simeq \tau_{E}$, the energy confinement time. Admittedly, if $\left\langle R \widehat{e}_{\phi} \cdot \vec{\nabla} \cdot \overleftrightarrow{\Pi}\right\rangle$ were taken to be the neoclassical viscous tensor, the corresponding $\tau_{\phi}$ would be a factor of 100 larger. For the parallel stress tensor, the neoclassical theory would suggest $\langle\vec{B} \cdot \vec{\nabla} \cdot \stackrel{\leftrightarrow}{\Pi}\rangle=\nu_{\|} n_{\mathrm{i}} m_{\mathrm{i}} K(\psi)\left\langle B^{2}\right\rangle$, where $\nu_{\|}$is the parallel viscosity. In the banana regime, $\nu_{\|} \sim \sqrt{\varepsilon} / \tau_{\mathrm{i}}$, where $\varepsilon$ is the aspect ratio and $\tau_{\mathrm{i}}$ is the ion collision time. This assumption will then lead to

$$
u_{\mathrm{p}} \simeq \frac{\tau_{\mathrm{i}}\left\langle\vec{B} \cdot \vec{F}^{\mathrm{rf}}\right\rangle}{\sqrt{\varepsilon} n_{\mathrm{i}} m_{\mathrm{i}}\left\langle B^{2}\right\rangle} B_{\mathrm{p}}
$$

We shall see that the kinetic treatment of the problem gives a similar result.

\section{KINETIC APPROACH TO THE PARALLEL FLOW PROBLEM}

In the toroidally rotating reference frame in which the radial electric field $E_{\mathrm{r}}=0$ and in the absence of angular acceleration $\partial \omega_{0} / \partial t=0$, the leading order gyrokinetic equation in poloidal gyroradius expansion is

$$
v_{\|} \widehat{b} \cdot \vec{\nabla} f=Q^{\mathrm{rf}}(f)+C(f),
$$

where $C(f)$ denotes the Coulomb collision operator and $Q^{\mathrm{rf}}(f)$ is given in Eq. (2). By taking the density moment of Eq. (10), it is straightforward to see that $\vec{u}=K(\psi) \vec{B}$ in the toroidally rotating frame. Consider the case that $f$ is close to Maxwellian, $f \simeq f_{M}+h$. Linearizing Eq. (10) about $f_{M}$ we have

$$
v_{\|} \widehat{b} \cdot \vec{\nabla} h-C_{\mathrm{ii}}^{\ell}(h)=Q^{\mathrm{rf}}\left(f_{\mathrm{M}}\right)
$$

By solving Eq. (11), the poloidal flow function can be evaluated as $n_{\mathrm{i}} K(\psi)$ $=\left\langle\int d^{3} v v_{\|} B h\right\rangle /\left\langle B^{2}\right\rangle$ without involving the stress tensor. Moreover, we may apply the adjoint techniques familiar in the study of if current drive to calculate $K(\psi)$ by introducing the parallel flow response function $g$ which satisfies the adjoint equation:

$$
-v_{\|} \widehat{b} \cdot \vec{\nabla} g-C_{\mathrm{ii}}^{\ell}(g)=\frac{v_{\|} B}{\left\langle B^{2}\right\rangle} f_{\mathrm{M}} .
$$

In terms of the function $g, n_{\mathrm{i}} K(\psi)=\left\langle\int d^{3} v g f_{\mathrm{M}}^{-1} Q^{\mathrm{rf}}\right\rangle$.

In the banana regime and the large aspect ratio limit, an approximate analytic solution of Eq. (12) is possible:

$$
g(v, \lambda)=-z v f_{M} \Phi(\lambda)\left(\frac{\tau_{\mathrm{i}}}{f_{\mathrm{t}}}\right)[1+O(\sqrt{\varepsilon})]
$$




$$
\Phi(\lambda)=\frac{\theta\left(\lambda_{c}-\lambda\right)}{2 f_{c}} \int_{\lambda}^{\lambda_{c}} \frac{d \lambda^{\prime}}{\left\langle\left(1-\lambda^{\prime} B\right)^{1 / 2}\right\rangle},
$$

with $\lambda_{c}=1 / B_{\max }$, where $z$ is a numerical constant of order $1, f_{\mathrm{t}}$ and $f_{\mathrm{c}}$ are, respectively, the effective trapped and circulating particle fraction, $f_{\mathrm{t}} \approx 1.46 \sqrt{\varepsilon}$. The presence of the factor $\left(\tau_{\mathrm{i}} / f_{\mathrm{t}}\right)$ in Eq. (13) indicates that the parallel drag is due to the collisions between the passing and trapped ions. The linear dependence of $g$ on $v$ reflects the fact that the parallel relaxation time is independent of velocity, in contrast to a dependence of $v^{3}$ in the case of electron current drive. This independence may be understood as follows: physically, a test passing ion, on the average, loses the memory of its initial velocity due to frequent collisions with other passing ions before it has a chance to collide with the trapped ions. Using Eq. (13), we find

$$
K(\psi)=\frac{-z \tau_{\mathrm{i}}}{n_{\mathrm{i}} f_{\mathrm{t}}}\left\langle\int d^{3} v v \Phi(\lambda) Q^{\mathrm{rf}}\right\rangle
$$

Furthermore, with the fact that $v \Phi(\lambda) \approx-v_{\|} B /\left\langle B^{2}\right\rangle$, we essentially recover the result of Eq. (9) for the driven poloidal rotation.

In summary, we have presented a theoretical framework, including moment and kinetic approaches, for considering if effects on plasma rotation in toroidal geometry. In the moment approach, the concept of rf forces and phenomenological relaxation times were introduced. The kinetic theory showed that a constant relaxation time model (independent of velocity) should be applicable in the case of parallel (poloidal) rotation. Quantitative studies of rf-induced rotation based on the present theoretical formulation will be relegated to a future work.

The authors wish to thank F.L. Hinton for many useful discussions. This is a report of work sponsored by the U.S. Department of Energy under Contract No. DE-AC03-89ER51114.

\section{REFERENCES}

[1] A.D. Turnbull et al., "Wall Stabilization of Rotating High $\beta$ Discharges in DIII-D," General Atomics Rep. GA-A21819 (1994).

[2] H. Biglari et al., Phys. Fluids B 2, 1 (1990).

[3] M.N. Rosenbluth and F.L. Hinton, "Plasma Rotation Driven by Alpha Particles in a Tokamak Reactor," submitted to Nuclear Fusion (1995).

[4] V.S. Chan and S.C. Chiu, Phys. Fluids B 5, 3590 (1993).

[5] The current source $\vec{\jmath}^{\text {ex }}$ can be used to model the current due to prompt loss of $\alpha$ particles in reactors or if excited ions (see Ref. 3 ). 